\title{
Nexo: Una herramienta para la visualización y análisis de indicadores QoS y QoE móviles
}

\section{Nexo: A tool for the visualization and analysis of mobile QoS and QoE indicators}

\author{
Ariel Machini ${ }^{1}$, Juan Enriquez ${ }^{2}$, Sandra Casas ${ }^{3}$ \\ 1arielmachini@protonmail.com, ${ }^{2}$ jenriquez@unpa.edu.ar, ${ }^{3}$ sicasas@conicet.gob.ar \\ GISP, Instituto de Tecnología Aplicada, Unidad Académica de Río Gallegos \\ Universidad Nacional de la Patagonia Austral, Río Gallegos, Argentina
}

Recibido: 30/03/2020. Aceptado: 11/09/2020

\begin{abstract}
RESUMEN
Con el creciente uso de las tecnologías móviles, resulta cada vez más importante tanto para los desarrolladores de aplicaciones como para prestadores de servicios brindar una óptima calidad a sus usuarios. En la actualidad, existen diversas herramientas que permiten visualizar los distintos indicadores de calidad que impactan en la experiencia del usuario; Sin embargo, los conceptos de Calidad de Servicio (QoS) y Calidad de Experiencia (QoE) usualmente son estudiados por separado, lo cual hace que el análisis completo de la calidad sea una tarea difícil. Es por esto que, en este proyecto, se presenta Nexo: una herramienta que permite recolectar indicadores de calidad (tanto QoS como QoE) en aplicaciones móviles, así como también clasificarlos y visualizarlos de manera gráfica con la finalidad de establecer vínculos entre estos. Para comprobar el correcto funcionamiento de Nexo, se llevó a cabo un caso de estudio en el que se recopilaron diversos indicadores QoS y QoE de una aplicación real (AntennaPod) y, posteriormente, dichos indicadores fueron analizados mediante la generación de distintos gráficos con la herramienta. A través de este caso de estudio, se pudo validar la relación entre la calidad del servicio y la calidad de la experiencia del usuario.
\end{abstract}

Palabras clave: Indicadores; Calidad; QoS; QoE; Tecnologías móviles.

\begin{abstract}
With the growth in the usage of mobile technologies, it is increasingly important for both application developers and service providers to provide optimum quality to their users. Currently, there are several tools that allow to visualize the different quality indicators that impact the user experience; However, the concepts of Quality of Service (QoS) and Quality of Experience (QoE) are often studied separately, which makes the complete analysis of quality a difficult task. That is why, in this project, Nexo is presented: a tool that allows quality indicators (both QoS and QoE) to be collected in mobile applications, as well as to classify and visualize them graphically in order to establish links between them. To verify the correct operation of Nexo, a case study in which various QoS and QoE indicators were collected from a real application (AntennaPod) was carried out and, later, those indicators were analyzed by the generation of different graphics with the tool. Through this case study, the relationship between quality of service and quality of user experience could be validated.
\end{abstract}

Keywords: Indicators; Quality; QoS; QoE; Mobile technologies. 


\section{INTRODUCCIÓN}

En la actualidad, dispositivos móviles tales como teléfonos inteligentes y tabletas son cada vez más populares y potentes. Los servicios y aplicaciones móviles se encuentran expuestos a una diversidad de factores contextuales, tales como la limitación de recursos (de memoria y CPU), un bajo porcentaje de batería en el dispositivo que los utiliza o canales físicos poco fiables y con ancho de banda limitado (Luo y Shyu, 2011; Devashish, 2013; Hefeeda y Hsu, 2010), que no solo afectan su desempeño (Calidad de Servicio o QoS), sino que también a la satisfacción de los usuarios finales (Calidad de Experiencia o QoE).

La degradación en la calidad que brindan los servicios y/o aplicaciones móviles es una de las causas por las cuales los usuarios finales deciden abandonarlos o cambiarlos. Es por esta razón, que la calidad es una preocupación tanto para los prestadores de servicios como para los desarrolladores de aplicaciones móviles.

La calidad es una problemática de especial interés para diversos actores (operadoras de servicios, desarrolladores de aplicaciones, usuarios finales y comunidad científicatecnológica). En particular, en la región de América de Sur y Caribe, los informes de Economía Móvil (GSMA, 2016; GSMA, 2017) señalan que la problemática de la calidad (QoS) y su percepción en los usuarios (QoE) se reconocen como una barrera para el acceso digital móvil y como una propiedad que a los usuarios les importa más que el precio.

Los estudios realizados en torno a la QoS (Quality of Service) y a la QoE (Quality of Experience) sobre distintos servicios y aplicaciones móviles buscan brindar una comprensión más profunda acerca de las percepciones del usuario final, planteando numerosas posibilidades de interés tanto para investigadores como para los distintos sectores de la industria móvil.

La recolección y la visualización de indicadores QoS y QoE móvil aportan información de utilidad para mejorar la calidad que brinda un servicio/aplicación a sus usuarios. Es por esto que, a partir de los indicadores que se recolectan (por lo general grandes volúmenes), resulta necesario llevar a cabo análisis tanto generales como puntuales de manera ágil y sencilla; por esta razón, la visualización gráfica de dichos indicadores surge como una alternativa válida. Sin embargo, el soporte a este tipo de funcionalidades en la actualidad es limitado y restringido: Por un lado, el análisis de la calidad se realiza, por lo general, de manera fragmentada, y por ello las herramientas recolectan indicadores de QoS o de QoE; por otro lado, la visualización gráfica integral de los indicadores recolectados constituye una funcionalidad rara vez utilizada.

Para los prestadores de servicios y desarrolladores de aplicaciones móviles resulta conveniente contar con herramientas para clasificar, analizar y visualizar los indicadores QoS y QoE recolectados de manera eficiente. De esta manera, se facilitan los cambios de configuraciones en la infraestructura de la red o de las aplicaciones para mejorar la experiencia de los usuarios finales.

Adicionalmente, otra debilidad observada es que las interacciones entre indicadores QoS y sus efectos en la QoE todavía no se conocen bien, así como no hay una metodología estandarizada que mapee de manera directa y cuantitativa QoS a QoE. La mayor parte de los métodos de mapeo son sólo enfoques parciales (Alreshoodi y Woods, 2013). 
En este trabajo se presenta Nexo, una herramienta de soporte para la visualización de indicadores QoS y QoE que permitirá al desarrollador analizar las relaciones que pueden existir entre estos. Con objeto de probar el correcto funcionamiento de Nexo, se desarrolló un caso de estudio para recolectar indicadores QoS y QoE mediante una aplicación móvil utilizando la librería Q2M (Machini et al., 2018), la cual fue desarrollada por este grupo de investigación en un trabajo anterior.

Este trabajo se encuentra organizado de la siguiente manera: En la Sección 2 se explica la metodología aplicada para el desarrollo del trabajo así como también las tecnologías que se utilizaron; En la Sección 3 se discute sobre los trabajos relacionados; En la Sección 4 se definen métricas e indicadores QoS y QoE y también se detalla brevemente la librería Q2M; En la Sección 5 se detalla sobre el diseño y la implementación de Nexo; En la Sección 6 se expone el caso de estudio llevado a cabo y en la Sección 7 se desarrollan las conclusiones y el trabajo a futuro.

\section{Metodología}

El enfoque metodológico aplicado para el desarrollo de este trabajo corresponde a DSR (Design Science Research) (Peffers et al., 2007), en tanto se centra en la búsqueda de soluciones centradas en los problemas y resulta adecuado para áreas de sistemas de información y/o tecnologías de la información.

Este enfoque se dirige al diseño y producción de artefactos, tales como constructos, modelos, métodos e instanciaciones. En particular, este trabajo propone una instanciación (muestran que constructos, modelos o métodos pueden ser implementados, como sistemas, prototipos e implementaciones). En la Tabla 1, se presenta cómo se ha descompuesto el proceso de investigación empleado, siguiendo DSR.

Tabla 1 - Proceso de investigación aplicado

\begin{tabular}{|c|c|c|c|}
\hline \multicolumn{4}{|c|}{ DSR: Enfoque orientado a problemas } \\
\hline $\begin{array}{l}\text { 1. Identificación del } \\
\text { problema }\end{array}$ & 2. Objetivos de la solución & 3. Desarrollo & 4. Evaluación \\
\hline $\begin{array}{l}\text { La calidad móvil (QoS y } \\
\text { QoE) se analiza y evalúa } \\
\text { de manera fragmentada, } \\
\text { generando información } \\
\text { incompleta de las causas } \\
\text { que afectan la experiencia } \\
\text { de usuario. }\end{array}$ & $\begin{array}{l}\text { Desarrollar una } \\
\text { herramienta que permita } \\
\text { el análisis y evaluación de } \\
\text { la calidad móvil de } \\
\text { manera integral. }\end{array}$ & $\begin{array}{l}\text { Implementación de una } \\
\text { herramienta para entornos } \\
\text { Android que registra } \\
\text { indicadores y métricas de } \\
\text { QoE y QoS a nivel } \\
\text { usuario-app y dispositivo } \\
\text { y procesa y visualiza la } \\
\text { información de manera } \\
\text { centralizada. }\end{array}$ & $\begin{array}{l}\text { Ejecución de un caso de } \\
\text { estudio que prueba las } \\
\text { distintas funciones de la } \\
\text { herramienta. }\end{array}$ \\
\hline
\end{tabular}

Varios procesos para DSR han sido propuestos; se hizo enfoque en las fases comunes a todos ellos, en los cuales se han aplicado técnicas mixtas de investigación.

a) Identificar el problema para el que se busca una solución: A partir de la revisión de la bibliografía disponible, se definió el problema. Con estas características, fue posible inferir objetivos y requisitos para el diseño y desarrollo de la solución, basada en el desarrollo de una herramienta. 
b) Diseñar y construir el artefacto que representa una solución al problema: En esta segunda etapa se desarrolló una herramienta cliente/servidor, a la cual hemos denominado Nexo. Para ello, se han utilizado distintas tecnologías:

- Principalmente, la librería Q2M (descrita en la Sección 4).

- XML (de eXtensible Markup Language): Lenguaje de marcado de propósito general, similar a HTML. A diferencia de otros lenguajes de marcado, XML no está predefinido, por lo que permite definir etiquetas propias ("Introducción a XML", 2019, párr. 1). En lo que a Nexo respecta, se hace uso del lenguaje XML para registrar la información de los indicadores recolectados por Q2M debido a la flexibilidad que este permite y a lo fácil que resulta procesar estos documentos (XML) en diversos lenguajes de programación.

- REST (de REpresentational State Transfer): Arquitectura que permite utilizar operaciones HTTP (tales como GET o PUT) para leer y alterar información. Diversos tipos de sistemas pueden actuar como servidores para este protocolo, incluyendo tanto aplicaciones específicas de dominio como sistemas de almacenamiento generales (Hawke, 2011). En Nexo, se emplea para recepcionar los indicadores recolectados por Q2M.

- Apache ${ }^{1}$ : Servidor HTTP de código abierto y multiplataforma, el cual se utiliza para mantener el visualizador web de Nexo en funcionamiento. Apache dispone de características altamente configurables y es el software de servidor web más utilizado ("Market Position Report of Top 5 Web Servers", 2020).

- MySQL: Sistema de gestión de bases de datos relacional que se utiliza para almacenar toda la información sobre indicadores que se recepciona a través del API REST.

- pChart $^{2}$ : Librería PHP que permite crear una diversidad de gráficos del lado del servidor. Se utiliza en el visualizador web de Nexo para generar gráficos a partir de los indicadores almacenados en la base de datos MySQL. Para este desarrollo, se empleó una versión modificada $^{3}$ de pChart compatible con PHP 7.

c) Evaluar el artefacto: Para la evaluación del artefacto (de la herramienta, Nexo), se ha llevado a cabo un caso de estudio que permitió comprobar el correcto funcionamiento de las distintas características implementadas. En el mismo se han definido los objetivos, elementos del estudio y un registro estructurado de los datos obtenidos.

\section{TRABAJOS RELACIONADOS}

En la literatura no se encuentran muchos trabajos directamente relacionados al tema de investigación propuesto. Sin embargo, existen proyectos que investigan diferentes aspectos involucrados en la presente investigación y se describen brevemente a continuación:

Relationship between Quality-of-Service and Quality-of-Experience for Public Internet Service (Khirman y Henriksen, 2002). En este trabajo se utilizó la metodología Semantic Traffic Analysis (desarrollada por Narus Inc.) para medir la QoE del usuario con el objetivo de establecer relaciones entre QoS y QoE. En los análisis efectuados en este proyecto, se estudió la relación entre la puntuación del usuario con diversas métricas QoS (ancho de banda, latencia y tiempo de entrega del objeto).

\footnotetext{
${ }^{1} \mathrm{https}: / /$ httpd.apache.org

${ }^{2}$ http://www.pchart.net

${ }^{3}$ https://github.com/bozhinov/pChart2.0-for-PHP7
} 
QoE-aware QoS Management (Agboma y Liotta, 2008). En este proyecto se empleó una técnica de modelado estadístico que correlaciona parámetros QoS con estimaciones de las percepciones de la QoE con la finalidad de identificar el grado de influencia de cada parámetro de QoS en la percepción del usuario.

A Generic Quantitative Relationship between Quality of Experience and Quality of Service (Fiedler, Hossfield y Tran-Gia, 2010). En este proyecto se propuso una fórmula genérica en la que diferentes parámetros de QoE y QoS están conectados a través de una relación exponencial, la cual llaman "hipótesis IQX". La fórmula propuesta relaciona los cambios en la QoE con respecto a la QoS con el nivel actual de QoE. En las pruebas realizadas en el marco de este proyecto, se abarcan diversas métricas QoS (jitter, pérdida de paquetes y reordenamiento) y QoE (latencia percibida por el usuario y puntuación del usuario).

A Study on a QoS/QoE Correlation Model for QoE Evaluationon IPTV Service (Kim y Choi, 2010). En este proyecto se desarrolló un modelo de correlación QoE/QoS para la evaluación de la QoE en IPTV. Las métricas QoS que se contemplaron en los casos de prueba llevados a cabo en este proyecto son el ancho de banda, el burst level, el jitter, la latencia y la pérdida de paquetes. Dichas pruebas se realizaron con distintas configuraciones de servicios de televisión.

Radar Chart: Scanning for Satisfactory QoE in QoS Dimensions (Chang et al., 2012). En este proyecto se desarrolló una herramienta para exponer las relaciones existentes entre distintas métricas QoS y la QoE haciendo uso de gráficos de radar. Este proyecto abarca tanto métricas QoS (ancho de banda, pérdida de paquetes y retardo) como QoE (Mean Opinion Score), y se realizan pruebas sobre aplicaciones de escritorio tales como Skype o Google Talk.

Prometheus: Toward Quality-of-Experience estimation for mobile apps from passive network measurements (Aggarwal et al., 2014). En este proyecto se desarrolló Prometheus, un sistema para el monitoreo y la predicción de la QoE en aplicaciones de video bajo demanda y VoIP reales. Consiste en implementaciones tanto en el dispositivo móvil (cliente) como en el operador del servicio, y estima las métricas de QoE que abordan los desafíos específicos de los operadores celulares. No requiere control sobre los servicios de la aplicación de destino y efectúa las predicciones de la QoE utilizando únicamente medidas de red pasivas.

QoE Doctor: Diagnosing mobile app QoE with automated UI control and cross-layer analysis (Chen et al., 2014). En este proyecto se desarrolló una herramienta para el análisis y el monitoreo de la QoE en aplicaciones móviles, sin la necesidad de acceder al código fuente de estas. Consiste en la implementación de una aplicación móvil con la finalidad de analizar las principales causas de la degradación de la QoE en las capas de aplicación, transporte, red y enlace de radio celular. Dicha aplicación emplea técnicas de automatización de la interfaz de usuario para reproducir el comportamiento del usuario que se relaciona con la QoE; y mide la latencia percibida por el usuario directamente de los cambios en la interfaz de usuario.

YoMoApp: A tool for analyzing QoE of YouTube HTTP adaptive streaming in mobile networks (Wamser et al., 2015). YoMoApp es una aplicación de análisis y monitoreo para Android que reproduce con precisión el comportamiento del servicio de YouTube para monitorear y almacenar pasivamente los indicadores clave de rendimiento de la transmisión de video adaptativa en teléfonos inteligentes. Puede monitorear eventos de usuario, nivel de búfer y calidad del video. Los datos recopilados se utilizan para analizar la QoE móvil que provee YouTube. 
ExBox: Experience management middlebox for wireless networks (Chakraborty et al., 2016). ExBox es un middlebox basado en un dispositivo de hardware y un software. Puede aprender la capacidad experiencial de una red a través de la estimación de la QoE y técnicas de aprendizaje automático (machine learning en inglés) diseñadas para entornos inalámbricos dinámicos.

User interface-level QoE analysis for Android application tuning (Lee y Cha, 2017). En este proyecto se desarrolló QX-probe, una herramienta comparativa y cuantitativa de análisis y monitoreo de la QoE que tiene como finalidad identificar los puntos de ajuste de la aplicación. Se basa en la ejecución de un servicio en el dispositivo móvil que se comunica con un servidor web. QX-probe define la latencia percibida por el usuario y el consumo de energía como factores críticos para el análisis de la QoE a nivel de interfaz de usuario. En el proyecto también se proporciona una herramienta web para el análisis de la QoE y la detección de puntos de ajuste.

Android Network measures (https://github.com/APISENSE/android-network-measures). Brinda herramientas para llevar a cabo mediciones de red (QoS) en Android mediante el uso de utilidades como ping, traceroute y descargas/subidas por TCP/UDP.

Android QoS SDK (https://github.com/RestComm/android-QoS). Provee métricas sobre QoS/QoE (tales como geolocalización, servicio de voz y mensajería) aplicables a diversos aspectos del sistema operativo Android, así como también sobre aplicaciones. Proporciona analíticas con el fin de permitir su incorporación nativa en aplicaciones móviles o SDK.

Fastah network kit API (https://getfastah.com). Facilita una librería para Android que tiene la finalidad de realizar estimaciones sobre la calidad de la red (QoS) de alta precisión. Mide la latencia y la congestión utilizando servidores propios de alto rendimiento.

QoE Probe for Android (https://github.com/farnazfotrousi/QoE-Probe-Android). Es una aplicación móvil Android que se puede integrar con otra aplicación para recolectar información relevante para la QoS y la QoE. La recolección de esta información tiene como objetivo estudiar las relaciones específicas entre las métricas de QoS y el impacto de la QoS en la QoE. Cabe destacar que también existe una variante para iOS de esta aplicación.

\section{MÉTRICAS E INDICADORES DE QOS Y QOE}

Una métrica (medida) es un valor numérico o nominal asignado a características o atributos de un ente computado a partir de un conjunto de datos observables y consistentes con la intuición. Otra posible definición de métrica es una correspondencia o mapeo de un dominio empírico (mundo real) a un mundo formal (matemático). Por lo tanto, una medida es un valor numérico o nominal asignado al atributo de un ente por medio de dicha correspondencia o mapeo. (DeMarco, 1986)

\section{Calidad de Servicio (QoS)}

Por lo general, la QoS es requerida cuando las aplicaciones son sensibles al retardo, y en redes donde la capacidad es un recurso limitado, como en la comunicación mediante una conexión de datos móviles. La QoS es la capacidad para proveer diferentes prioridades a diferentes 
aplicaciones/flujos de datos/usuarios, así como también la capacidad para garantizar cierto nivel de rendimiento para determinado flujo de datos (Svend y Jari, 1998).

La QoS brinda los siguientes beneficios: Le otorga a los administradores control sobre recursos de red y les permite administrar la red desde una perspectiva de negocio en lugar de hacerlo desde una perspectiva técnica; Asegura que aplicaciones sensibles al tiempo y que aplicaciones de misión crítica tengan los recursos que necesitan, permitiendo también el acceso a la red a otras aplicaciones; mejora la experiencia del usuario y reduce los costos al utilizar los recursos existentes eficientemente, de esta manera retrasando o reduciendo la necesidad de expansiones o mejoras (Gurwinder, 2011).

\section{Calidad de Experiencia (QoE)}

La QoE se refiere a la aceptabilidad general de una aplicación o servicio, de acuerdo a la percepción subjetiva del usuario final: Se refiere a cómo el usuario percibe la calidad. De esta manera la QoE incluye todos los aspectos de un sistema de extremo a extremo (cliente, terminal, red, infraestructura de servicios, etc.), donde la aceptabilidad general puede verse influenciada por las expectativas del usuario, así como también por el contexto (Kuipers et al., 2010).

Las métricas correspondientes a la QoE se pueden subdividir en dos tipos: objetivas y subjetivas. Las métricas subjetivas se encuentran relacionadas a la opinión del usuario, que evalúa la calidad de la aplicación o del servicio en base a su experiencia con la aplicación (Ickin, 2015), dependiendo su evaluación de factores subjetivos tales como el contexto o las expectativas que tiene el usuario de la aplicación. Con respecto a las métricas objetivas, se destaca su carácter sistemático, exacto y repetible, y representan diversas propiedades como el tiempo de presentación de los datos, el consumo de los datos móviles, el consumo de energía, la interfaz de usuario y la presentación del contenido (Ickin, 2015).

\section{Librería Q2M}

La librería Q2M ${ }^{4}$ (Machini et al., 2018), desarrollada en un proyecto de investigación anterior, permite recolectar indicadores de calidad QoS y QoE desde una aplicación Android. Q2M se construyó para Android ya que es un sistema operativo de código abierto y también es el más utilizado 5 ("Smartphone Market Share", 2019) en dispositivos móviles.

En la Tabla 2 se describen brevemente los indicadores que abarca dicha librería.

Tabla 2 - Indicadores QoE y QoS de la librería Q2M.

\begin{tabular}{|c|l|c|}
\hline \multicolumn{1}{|c|}{ Indicador } & \multicolumn{1}{|c|}{ Descripción } & Tipo \\
\hline Batería cargando & Estado de carga del teléfono (cargando/sin cargar). & QoE \\
\hline Brillo de la pantalla & Porcentaje de brillo de la pantalla del teléfono del usuario. & QoE \\
\hline Conexión a una red & $\begin{array}{l}\text { Indicador de conexión del teléfono a una red (independientemente si tiene } \\
\text { conexión a Internet o no). }\end{array}$ & QoE \\
\hline Intensidad de la señal & Intensidad de la señal de la conexión en uso en dBm. & QoE \\
\hline
\end{tabular}

\footnotetext{
${ }^{4}$ https://github.com/gispunpauarg/Q2M

${ }^{5}$ De acuerdo a la fuente referenciada, en 2019 Android logró ocupar el 87\% de la cuota de mercado.
} 


\begin{tabular}{|c|c|c|}
\hline $\begin{array}{l}\text { Latencia percibida por } \\
\text { el usuario }\end{array}$ & $\begin{array}{l}\text { Tiempo desde que el usuario dispara cierto evento hasta que recibe una } \\
\text { respuesta. }\end{array}$ & QoE \\
\hline Luz ambiental & Nivel de luz del entorno en el que se encuentra el usuario en lx (lux). & QoE \\
\hline $\begin{array}{l}\text { Porcentaje de carga de } \\
\text { la batería }\end{array}$ & Porcentaje de carga actual de la batería del teléfono. & QoE \\
\hline Proximidad & $\begin{array}{l}\text { Distancia entre el dispositivo y el objeto más próximo a su sensor de } \\
\text { proximidad. }\end{array}$ & QoE \\
\hline Tipo de conexión & $\begin{array}{l}\text { Tipo de conexión (Wi-Fi, 4G, 3G, GPRS, etcétera) a la que está conectado el } \\
\text { dispositivo del usuario mientras utiliza la aplicación. }\end{array}$ & QoE \\
\hline Uso de memoria & $\begin{array}{l}\text { Cantidad de MB o porcentaje de la memoria RAM del teléfono que está en } \\
\text { uso. }\end{array}$ & QoE \\
\hline $\begin{array}{l}\text { Uso del procesador por } \\
\text { parte de la aplicación }\end{array}$ & Porcentaje de uso del procesador que está utilizando la aplicación. & QoE \\
\hline Jitter & $\begin{array}{l}\text { Variación del retardo de la red en la comunicación a una dirección IP } \\
\text { determinada. }\end{array}$ & QoS \\
\hline Latencia & $\begin{array}{l}\text { Tiempo de respuesta transcurrido en la comunicación a una dirección IP } \\
\text { determinada. }\end{array}$ & QoS \\
\hline Pérdida de paquetes & $\begin{array}{l}\text { Porcentaje de pérdida de paquetes en la comunicación con una dirección IP } \\
\text { determinada. }\end{array}$ & QoS \\
\hline Puntuación del usuario & $\begin{array}{l}\text { Valor que determina el usuario de la aplicación para la experiencia que está } \\
\text { teniendo con esta. Puede elegir entre "Mala", "Regular" y "Buena". }\end{array}$ & QoE \\
\hline
\end{tabular}

Mediante los métodos que ofrece la librería Q2M, el desarrollador puede registrar diversos indicadores de calidad.

Para esto, el desarrollador debe definir qué indicadores necesita recopilar y, posteriormente, introducir las llamadas a los métodos de la librería que correspondan a cada uno de los indicadores en las secciones del código fuente de la aplicación donde sea necesario. Cada vez que en la aplicación se ejecute el código donde se encuentran las llamadas a los métodos de Q2M, se computarán los indicadores considerados y estos serán registrados en un archivo XML.

En el marco de este proyecto, la librería Q2M será modificada para que envíe esta información a un servidor en donde será procesada.

\section{NEXO}

El funcionamiento, diseño e implementación de Nexo se basan en dos partes: En el lado del cliente, la librería Q2M, la cual se implanta a una aplicación móvil a partir de la cual se requieren obtener indicadores de calidad; En el lado del servidor, un visualizador web, que almacena, procesa y presenta la información sobre los indicadores.

Esto se puede representar mediante cuatro etapas, las cuales se pueden contemplar en la Figura 1: recolección, envío, registro y clasificación y análisis. 


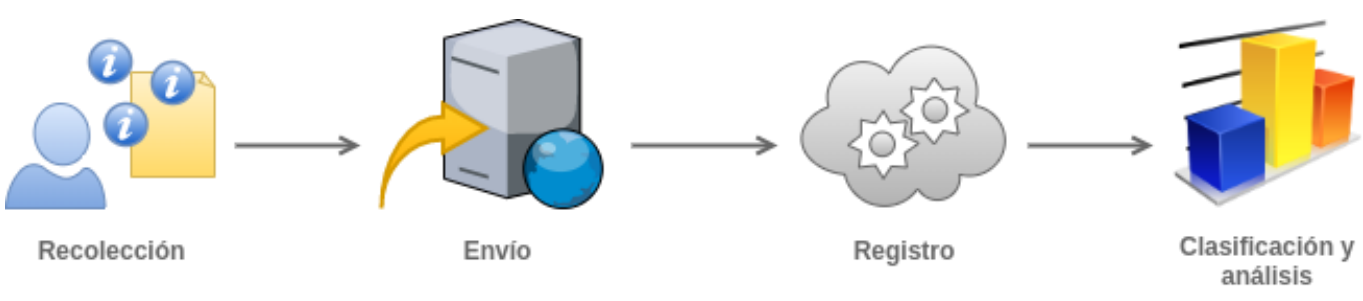

Figura 1 - Etapas que describen el funcionamiento de Nexo.

El cliente se encarga de las dos primeras etapas funcionales. La etapa de recolección, en la que se registrarán indicadores de calidad y se almacenarán en un archivo XML para cada usuario de la aplicación (todo esto mediante las funcionalidades que brinda la librería Q2M); y la etapa de envío en la que, a través de una red, los archivos XML con los indicadores previamente registrados se enviarán a un servidor periódicamente para su procesamiento.

El servidor se encarga de las dos etapas funcionales restantes. La etapa de registro, en la que los indicadores que reciba el servidor (agrupados en archivos XML) serán procesados y almacenados en una base de datos; y la etapa de clasificación y análisis en la que, mediante una interfaz web, los indicadores podrán clasificarse en función de algún criterio y podrán visualizarse en forma de gráficos.

Nexo permite guardar los indicadores recolectados mediante la librería Q2M en una base de datos, así como también su organización y presentación a través de gráficos para su subsiguiente análisis. Los componentes de esta herramienta y su funcionamiento se encuentran ilustrados en la Figura 2.

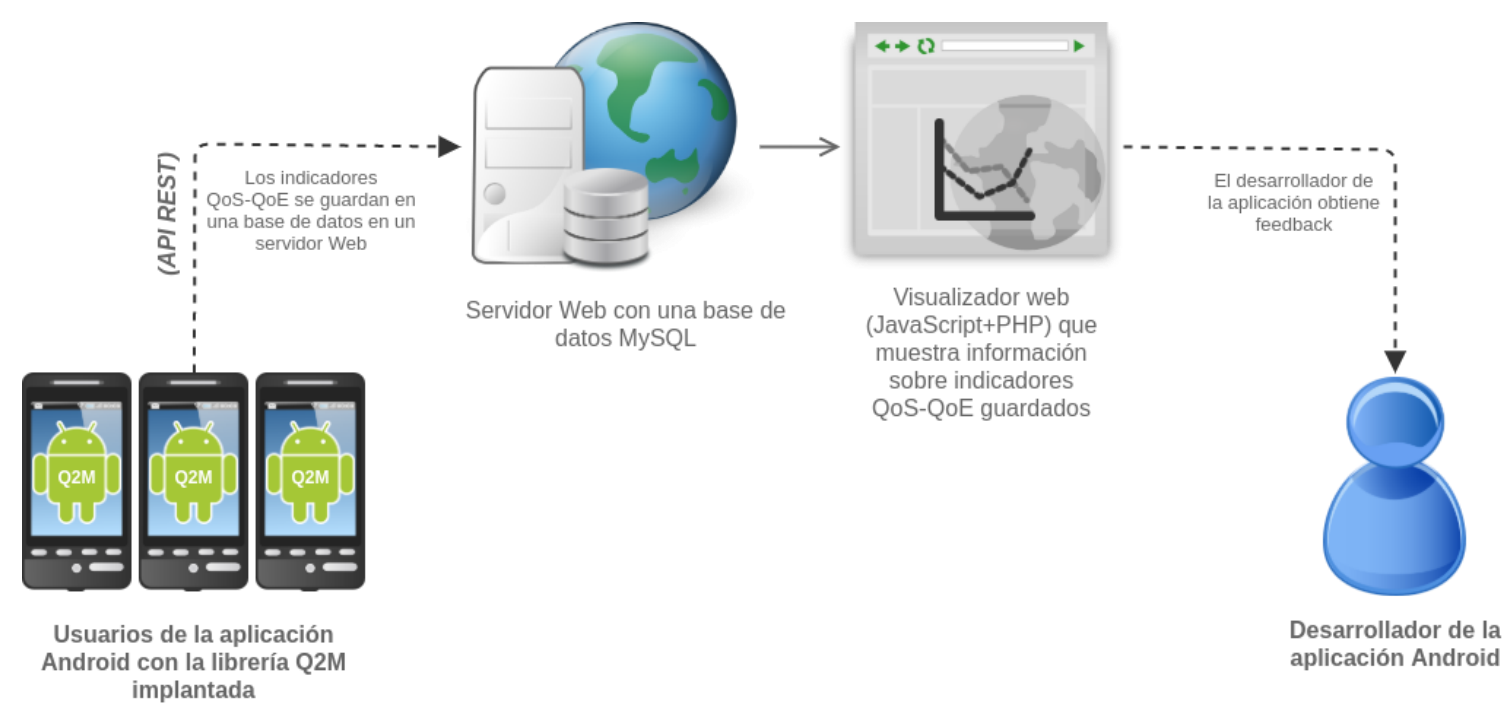

Figura 2 - Esquema sobre el funcionamiento de Nexo.

\section{Visualizador web}

El visualizador web de Nexo permite consultar los valores de los indicadores registrados de manera gráfica y amigable. Mediante la observación de estos datos, se pueden establecer relaciones entre los distintos indicadores y la calidad de la experiencia del usuario. Esta información permitirá al desarrollador conocer qué aspectos de la aplicación o servicio requieren cambios para que mejore dicha experiencia. 
Para generar los gráficos se utiliza pChart, una librería que permite construir una gran diversidad de gráficos con distintas configuraciones.

En la Figura 3, se puede observar la interfaz del visualizador web, donde se selecciona el usuario cuyos indicadores (ya sean QoS o QoE) se requieren analizar y comparar con la calidad de la experiencia de dicho usuario.

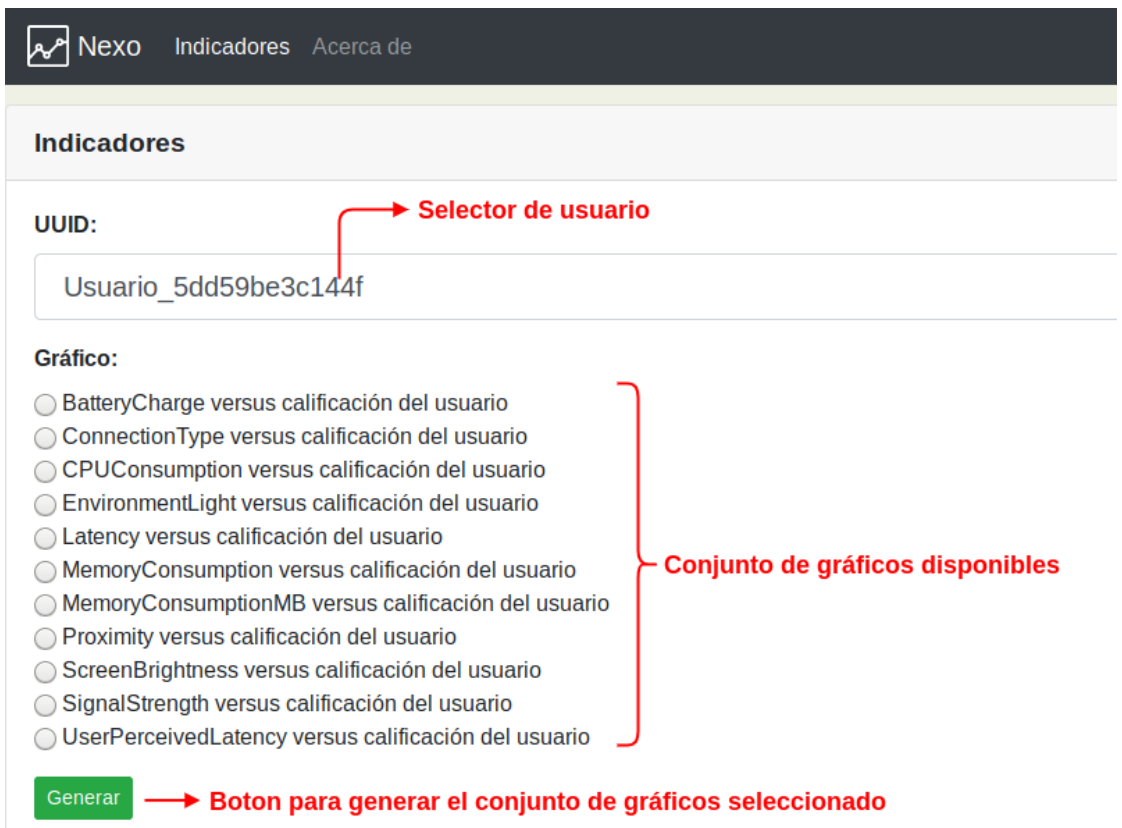

Figura 3 - Descripción de la interfaz web.

Una vez que se selecciona el usuario, se podrá generar una variedad de gráficos comparativos que facilitarán establecer relaciones entre los valores registrados para ciertos indicadores y el nivel de satisfacción expresado por el usuario. En la Figura 4 se muestra un conjunto de gráficos de ejemplo que contrasta la latencia con la calificación del usuario. 


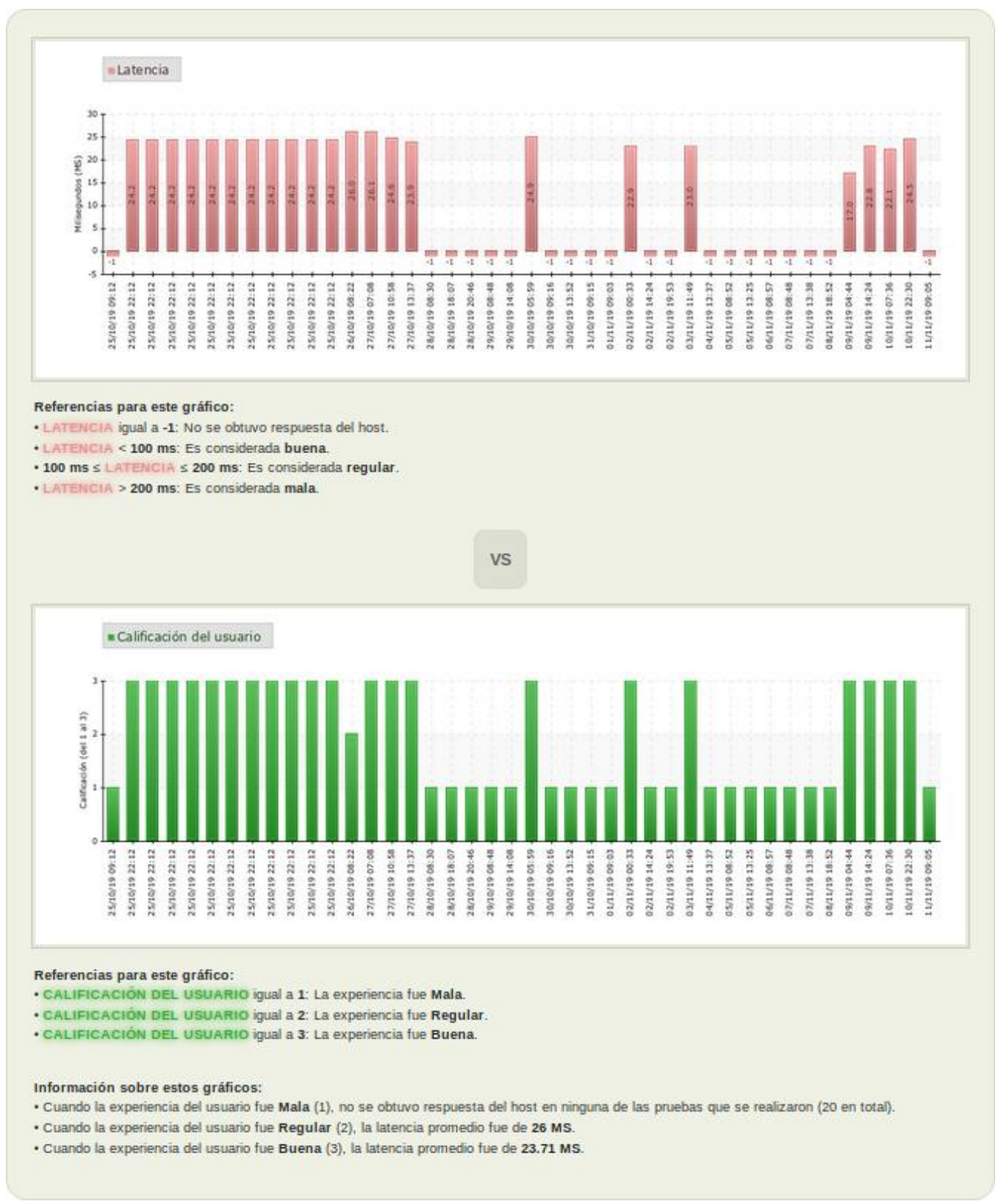

Figura 4 - Conjunto de gráficos de ejemplo (latencia versus calificación del usuario).

Debido al reducido tiempo disponible, la versión de Nexo presentada en este trabajo cuenta con ciertas limitaciones: No puede procesar todos los indicadores que recopila la librería Q2M (presentes en la Tabla 2); Sólo puede generar un reducido grupo de gráficos; Sólo puede generar gráficos de barras y sólo puede mostrar comparaciones entre dos métricas. Con respecto a la primera de las limitaciones, Nexo sólo puede procesar los siguientes indicadores: brillo de la pantalla, jitter, latencia, latencia percibida por el usuario, luz ambiental, porcentaje de carga de la batería, uso de memoria, uso del procesador por parte de la aplicación y puntuación o calificación del usuario.

\section{Caso de estudio: AntennaPod}

A fines de evaluar, probar y verificar el funcionamiento de la herramienta, se ha llevado a cabo un caso de estudio generando e integrando pruebas para la recolección de indicadores 
QoS y QoE sobre una aplicación móvil real. El usuario partícipe del caso de estudio utilizó AntennaPod ${ }^{6}$, una aplicación para descargar y escuchar podcasts a la que se le incorporó la librería Q2M para recolectar ciertos indicadores cada vez que requiere descargar un episodio de un podcast. En este caso en particular, los indicadores sobre los cuales se recolectó información son brillo de la pantalla, latencia, latencia percibida por el usuario, luz ambiental, porcentaje de carga de la batería, uso de memoria, uso del procesador por parte de la aplicación y puntuación o calificación del usuario ${ }^{7}$. Nótese que los detalles sobre estos indicadores están presentes en la Tabla 2.

A continuación (en la Tabla 3), se describe brevemente el perfil del usuario:

Tabla 3 - Perfil del usuario que formó parte del caso de estudio.

\begin{tabular}{|c|c|c|}
\hline \multicolumn{2}{|c|}{ Información básica } & Sexo \\
\hline Edad & Hombre & Ocupación \\
\hline 38 años & \multicolumn{2}{|c|}{} \\
\hline Detalles del dispositivo & Modelo & $\begin{array}{c}\text { Versión del sistema operativo } \\
\text { Android }\end{array}$ \\
\hline Marca & Galaxy S8 & 9 (Pie) \\
\hline Samsung &
\end{tabular}

\section{Análisis del caso de estudio}

Mediante las funcionalidades que proporciona el visualizador web de Nexo, se crearon diversos gráficos a partir de la información generada por el usuario presentado en la Tabla 3.

Con la finalidad de brindar contexto, en la Tabla 4 se muestran los valores promedio y/o moda ${ }^{8}$ observados en los gráficos generados para cada uno de los indicadores contemplados.

Tabla 4 - Valores promedio y/o moda de los indicadores recolectados.

\begin{tabular}{|c|l|}
\hline Indicador & \multicolumn{1}{|c|}{ Valor promedio y/o moda } \\
\hline Brillo de la pantalla & $63 \%$ \\
\hline Intensidad de la señal & $-75.18 \mathrm{dBm}$ \\
\hline Latencia & $24.86 \mathrm{~ms}$ \\
\hline Latencia percibida por el usuario & $\begin{array}{l}4.8 \text { segundos como valor promedio; 0 segundos como } \\
\text { moda }\end{array}$ \\
\hline Luz ambiental & 1103.72 lux \\
\hline Porcentaje de carga de la batería & $86 \%$ \\
\hline Proximidad & 8 centímetros \\
\hline
\end{tabular}

\footnotetext{
${ }^{6}$ https://github.com/AntennaPod/AntennaPod

${ }^{7}$ No se abarcaron todos los indicadores de calidad que puede recolectar la librería Q2M (Tabla 1) debido a una de las limitaciones de Nexo.

${ }^{8}$ La moda es el valor con más frecuente de una variable, o sea, el valor que más se repite en la población o muestra. (Rustom Jabbaz, 2012)
} 


\begin{tabular}{|c|l|}
\hline Tipo de conexión & LTE \\
\hline Uso de memoria & $68.46 \%(2518.49 \mathrm{MB})$ \\
\hline Uso del procesador por parte de la aplicación & $2.2 \%$ \\
\hline Calificación del usuario & $\begin{array}{l}2 \text { o regular como valor promedio; } 3 \text { o buena como } \\
\text { moda }\end{array}$ \\
\hline
\end{tabular}

Aunque Nexo permite recolectar una gran variedad de indicadores de calidad, para este caso de estudio en particular, se profundizará el análisis sobre la información que se registró sobre los indicadores latencia (QoS), latencia percibida por el usuario (QoE) y calificación del usuario ya que, al ser AntennaPod una aplicación para descargar (y escuchar) podcasts, se planteó la hipótesis de que la calidad de la experiencia que esta brinda estará atada en parte a la calidad de la conexión a internet que esté utilizando el usuario. A continuación, en la Figura 5, Figura 6 y Figura 7 se muestran los gráficos generados por el visualizador web para los indicadores antes mencionados.

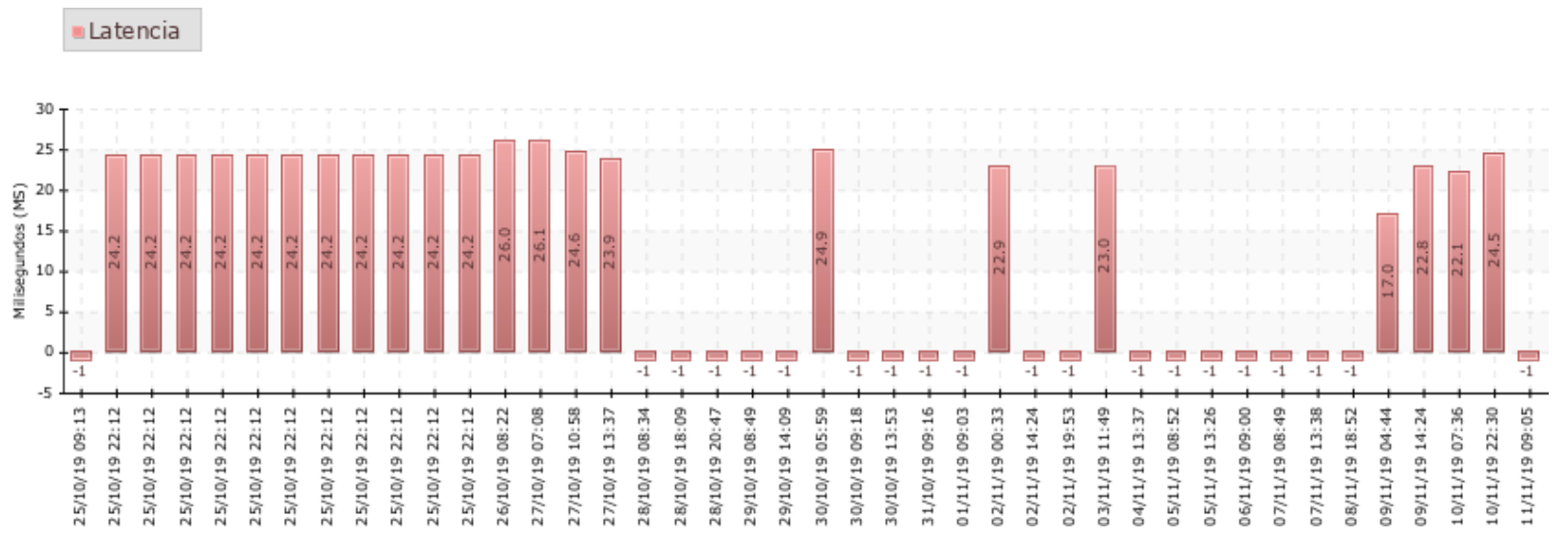

Figura 5 - Gráfico correspondiente a la latencia.

De acuerdo a las recomendaciones presentadas en ITU-T Y.1540 (ITU, 2002), a excepción de las pruebas en la que la aplicación no pudo comunicarse con el servidor, se registró una latencia aceptable: Como se indicó en la Tabla 4, el valor promedio fue de 24.86 ms.

- Latencia percibida por el usuario

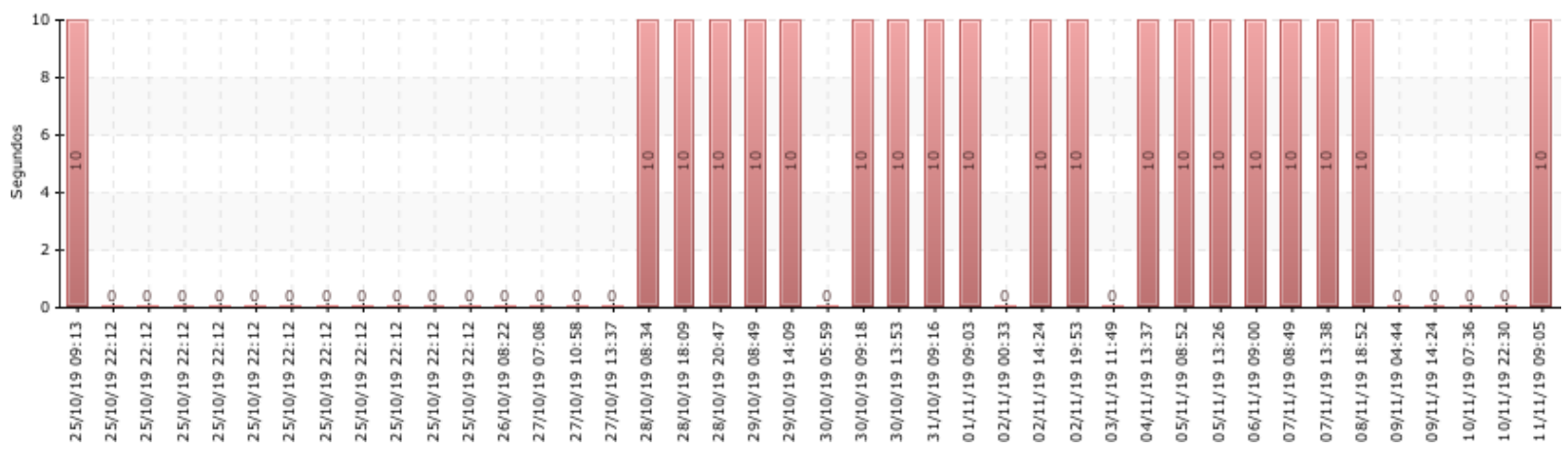

Figura 6 - Gráfico correspondiente a la latencia percibida por el usuario.

Comparando la información expuesta en la Figura 6 con la Figura 5, se puede llegar a la conclusión que, siempre que hubo un error de comunicación con el servidor (es decir, que no 
se obtuvo una respuesta por parte del host) se observó un incremento considerable (un valor constante de 10 segundos) en la latencia percibida por el usuario (indicador de QoE).

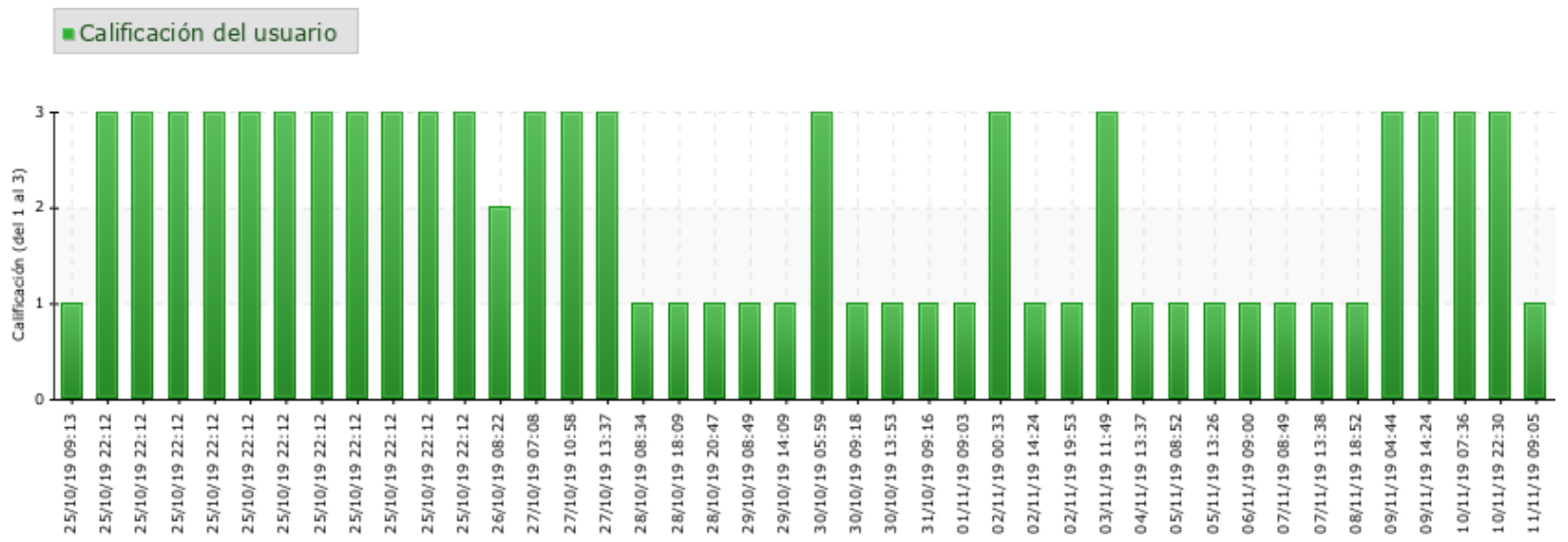

Figura 7 - Gráfico correspondiente a la calificación del usuario.

\section{CONCLUSIONES}

En este trabajo se presentó $\mathrm{Nexo}^{9}$, una herramienta que permite recolectar y visualizar gráficamente indicadores QoS y QoE para aplicaciones móviles, con la finalidad de que se puedan descubrir relaciones entre dichos indicadores fácilmente.

En el caso de estudio desarrollado en la Sección 6, tras analizar la información presentada por Nexo, se pudo observar que el usuario vivenció una buena experiencia con AntennaPod. Sin embargo, se descubrió que la calificación que otorgó dependió fuertemente de los indicadores latencia y latencia percibida por el usuario (estando este último indicador influenciado por la latencia). Es por esto que se puede afirmar que la hipótesis presentada anteriormente ha sido comprobada y, como argumentan diversos investigadores, existen indicadores QoS que pueden impactar directamente en la QoE percibida por el usuario; Es por esta razón, que un análisis integral de la calidad resulta conveniente (Kosinski et al., 2008; Kim et al., 2008; Moor et al., 2016; Nawrocki y Sliwa, 2016).

El trabajo futuro está orientado a reducir la cantidad de limitaciones que presenta la herramienta: ampliar los tipos de indicadores de calidad con los que Nexo puede tratar (principalmente: Pérdida de paquetes; Intensidad de la señal; Tipo de conexión y Conexión a una red. Todos estos indicadores se encuentran descritos en la Tabla 2), así como también añadir funcionalidad a la herramienta para que sea posible realizar contrastaciones más complejas entre indicadores (por ejemplo, entre indicadores QoS que están relacionados, como latencia versus jitter; o la comparación entre más de dos indicadores de calidad).

\section{REFERENCIAS}

AGGARWAL, V., HALEPOVIC, E., PANG, J., VENKATARAMAN, S. y YAN, H. (2014). Prometheus: Toward Quality-of-Experience estimation for mobile apps from passive network measurements. Proceedings of the 15th Workshop on Mobile Computing Systems and Applications, pp. 1-6.

ALRESHOODI, M. y WOODS, J. (2013). Survey on QoElQoS correlation models for multimedia services. arXiv preprint arXiv:1306.0221.

\footnotetext{
${ }^{9}$ Código fuente disponible públicamente en GitHub: https://github.com/gispunpauarg/Nexo
} 
CHAKRABORTY, A., SANADHYA, S., DAS, S. R., KIM, D. y KIM, K. H. (2016). ExBox: Experience management middlebox for wireless networks. Proceedings of the 12th International on Conference on emerging Networking EXperiments and Technologies, pp. 145-159.

CHEN, Q. A., LUO, H., ROSEN, S., MAO, Z. M., IYER, K., HUI, J., SONTINENI, K. y LAU, K. (2014). QoE Doctor: Diagnosing mobile app QoE with automated UI control and cross-layer analysis. Proceedings of the 2014 Conference on Internet Measurement Conference, pp. 151-164.

DEMARCO, T. (1986). Controlling Software Projects: Management, Measurement and Estimates. $1^{\circ}$ ed. Prentice Hall, Englewood Cliffs, Estados Unidos.

DEVASHISH, G. (2013). Mobile Computing. International Journal of Advanced Research in Computer Science and Software Engineering, vol. 3, nro. 9, pp. 846-855.

GSMA. The Mobile Economy Latin America and the Caribbean 2016. Recuperado el 5 de Marzo de 2020 de https://www.gsma.com/latinamerica/resources/mobile-economylatin-america-caribbean-2016.

GSMA. The Mobile Economy Latin America and the Caribbean 2017. Recuperado el 6 de Marzo de 2020 de https://www.gsma.com/latinamerica/resources/mobile-economylatin-america-caribbean-2017.

GURWINDER, P. (2011). QoS Modeling and Analysis using QML in Mobile Computing Environment (Tesis doctoral, Jayoti Vidyapeeth Women's University). Recuperado de https://shodhgangotri.inflibnet.ac.in/bitstream/123456789/1218/1/gurwinder\%20paul \%20kaurcorrected.pdf

HAWKE, S. (16 de Diciembre de 2011). REST. W3C. Recuperado de https://www.w3. org/2001/sw/wiki/REST

HEFEEDA, M. y HSU, C. H. (2010). Mobile video streaming in modern wireless networks. Proceedings of the 18th ACM international conference on Multimedia, pp. 1779-1780.

ICKIN, S. (2015). Quality of Experience on Smartphones: Network, Application, and Energy Perspectives (Tesis doctoral, Blekinge Institute of Technology). Recuperado de http://www.diva-portal.org/smash/get/diva2:833883/FULLTEXT02

IDC. Smartphone Market Share. Recuperado el 31 de Diciembre de 2019 de https://www.idc.com/promo/smartphone-market-share/os

ITU (2002). ITU-T Recommendation Y.1541. Series Y: Global information infrastructure and Internet protocol aspects. Internet protocol aspects - Quality of Service and network performance. Network performance objectives for IP-based services.

KIM, H., LEE, D., LEE, J., LEE, K., LYU, W. y CHOI, S. (2008). The QoE Evaluation Method through the QoS-QoE Correlation Model. Fourth International Conference on Networked Computing and Advanced Information Management, vol. 2, pp. 719-725.

KOSINSKI, J., NAWROCKI, P., RADZISZOWSKI, D., ZIELINSKI, K., ZIELINSKI, S., PRZYBYLSKI, G. y WNEK, P. (2008). SLA monitoring and management framework for telecommunication services. Fourth International Conference on Networking and Services (icns 2008), pp. 170-175. IEEE.

KUIPERS, F., KOOIJ, R., DE VLEESCHAUWER, D. y BRUNNSTRÖM, K. (2010). Techniques for Measuring Quality of Experience. Trabajo presentado en International Conference on Wired/Wireless Internet Communications, pp. 216-227. Springer, Heidelberg, Alemania.

LEE, S. y CHA, H. (2017). User interface-level QoE analysis for Android application tuning. Pervasive and Mobile Computing, vol. 40, pp. 382-396.

LUO, H. y SHYU, M. L. (2011). Quality of Service provision in mobile multimedia - a survey. Human-centric computing and information sciences, vol. 1, nro. 1, pp. 1-15. Springer. 
MACHINI, A., ENRIQUEZ, J. y CASAS, S. (2019). Q2M, una librería para computar métricas de calidad en aplicaciones móviles. Informes Científicos Técnicos - UNPA, vol. 11, nro. 2, pp. 1-17. https://doi.org/10.22305/ict-unpa.v11i2.783

MDN. Introducción a XML. Recuperado el 16 de Febrero de 2020 de https://developer.mozilla.org/es/docs/Web/XML/Introducción_a_XML

MOOR, K., KETYKO, I., JOSEPH, W., VERDEJO, J., PESSEMIER, T. y MAREZ, L. (2010). Evaluating Quality of Experience of mobile applications and services in a Living Lab setting. Mobile Networks and Applications, vol. 15.

NAWROCKI, P. y ŚLIWA, A. (2016). Quality of Experience in the context of mobile applications. Computer Science, vol. 17, nro. 3, pp. 371-385.

PEFFERS, K., TUUNANEN, T., ROTHENBERGER, M. A., y CHATTERJEE, S. (2007). A design science research methodology for information systems research. Journal of management information systems, vol. 24, nro. 3, pp. 45-77.

RUSTOM JABBAZ, A. (2012). Estadística descriptiva, probabilidad e inferencia: Una visión conceptual y aplicada. Facultad de Ciencias Agronómicas, Universidad de Chile.

SVEND, F. Y JARI, K. (1998). QML: A language for quality of service specification. HP Laboratories Technical Report, HPL-98-10.

$\mathrm{W}^{3}$ Techs. Market Position Report of Top 5 Web Servers. Recuperado el 17 de Febrero de $2020 \mathrm{de}$ https://w3techs.com/technologies/market/web_server

WAMSER, F., SEUFERT, M., CASAS, P., IRMER, R., TRAN-GIA, P. y SCHATZ, R. (2015). YoMoApp: A tool for analyzing QoE of YouTube HTTP adaptive streaming in mobile networks. 2015 European Conference on Networks and Communications (EuCNC), pp. 239-243. IEEE. 